\title{
Epidural opiate analgesia for acute pain relief
}

the greater ease of attenuating temporal summation acting at the neuronal membrane with the slowly conducting $\mathrm{C}$ fibre compared with the rapidly conducting A-delta fibre; different opioid receptors may be associated with different types of pain, and pharmacokinetic factors determining the amount of opioid reaching the receptors.

\section{Pharmacokinetics of epidural opioids}

Epidural opioids may be given as intermittent boluses or as a bolus/infusion combination. The pharmacokinetic profiles in blood, CSF and spinal cord of the differing opioids are complex and are related to the mode of administration (bolus or infusion), drug dose, lipid solubility, uptake by epidural fat and uptake by nonspecific lipid structures in the spinal cord. ${ }^{6}$ Morphine, a highly ionized and hydrophilic opioid, crosses the dura slowly appearing in CSF in 15 min with peak concentrations at 90 to 120 min. ${ }^{7}$ However, peak plasma concentrations were seen at $15 \mathrm{~min}$ and the plasma curves were similar to those seen after IM injection. Eighty per cent of the morphine is still present in the CSF four hours after injection and 50 per cent after $12 \mathrm{hr}^{8}{ }^{8}$ This supports the long latency and prolonged duration of analgesia after bolus doses of epidural morphine. The pharmacokinetic profile of a continuous epidural morphine infusion has been less investigated but was associated with adequate analgesia and undetectable serum levels of morphine in one study. ${ }^{72}$ In contrast, lipophilic (albeit ionized) opioids such as meperidine enter the CSF rapidly although plasma uptake for meperidine is slower than morphine. ${ }^{10}$ Highly soluble opioids such as fentanyl and sufentanil are rapidly absorbed into the vascular space when given as a bolus or infusion and may exert some of their analgesic effects systemically. ${ }^{11-15}$ There are no studies in humans on the neuraxial pharmacokinetics of highly lipid soluble opioids.

\section{Analgesic effects}

Postoperative pain

Dose requirements of epidural opioids relate to the sium channels, kappa receptors inhibit voltage-dependent calcium channels). In acute pain states, sharp pain (A-delta fibres) is less readily blocked than dull pain (C fibres). This may be related to the deeper location of A-delta fibres in the dorsal horn compared with $\mathrm{C}$ fibres;
Department of Anaesthesia, Toronto Gencral Hospital, University of Toronto, Toronto, Ontario 
surgical procedure, opioid used, site of catheter placement, use of bolus dose alone or bolus plus infusion, age, severity of concurrent illness, use of ancillary agents (e.g., local anaesthetic drugs) and interpatient variability in analgesic response. ${ }^{2-3} \mathrm{~A}$ comparison of examples of lipid insoluble opioids (e.g., morphine) and lipid soluble opioids (e.g., fentanyl, sufentanil) illustrates the different effects of these two classes when used in the epidural space.

\section{Morphine}

Although epidural morphine is widely used there are few dose-finding studies. For lower limb surgery lumbar bolus doses between 2 to $5 \mathrm{mg}$ are effective. ${ }^{16}$ Abdominal and thoracic procedures tend to require higher doses with lumbar catheters $(5-10 \mathrm{mg}) .^{17.18}$ Analgesic onset time tends to be slow taking up to $60 \mathrm{~min}$ to develop. ${ }^{16,19}$ Studies examining the siting of the catheter (lumbar vs thoracic) for thoracic analgesia have produced conflicting results ${ }^{20.21}$ although onset time for analgesia and dose requirements may be decreased with thoracic catheters. ${ }^{21}$ In addition, dose requirements may be decreased if epidural morphine is given preoperatively or very early during the surgical procedure. ${ }^{22-24}$ Prolonged analgesia has been noted to follow small doses $(3-5 \mathrm{mg})$ after Caesarean section. ${ }^{25,26}$ Morphine infusions set at approximately 0.1 to $0.2 \mathrm{mg} \cdot \mathrm{kg}^{-1}$ after a bolus of $2 \mathrm{mg}$ have been reported to be effective. ${ }^{9,27}$

\section{Lipid soluble opioids}

\section{FENTANYL}

Epidural fentanyl has been used for several types of postoperative pain, both as a bolus ${ }^{28-30}$ and as a bolus and infusion. ${ }^{14,15,31,32}$ Onset of analgesia is fairly rapid $(5-20$ $\mathrm{min}$ ) although of short duration (2-4 hr) if an infusion is not started. The requirement for large infusion doses $(1-2$ $\mu \mathrm{g} \cdot \mathrm{kg}^{-1} \cdot \mathrm{hr}^{-1}$ ) especially with lumbar catheters for thoracic pain ${ }^{14,15,33}$ may be related to the rapid uptake of epidural fentanyl in the blood stream ${ }^{14.15 .33}$ and uptake by non-specific binding sites in the spinal cord. ${ }^{6}$ Evidence is accumulating that epidural and intravenous fentanyl infusions may be equivalent in providing analgesia and have a similar incidence of side effects. ${ }^{13,15,34}$

\section{SUFENTANIL}

Epidural sufentanil has been used to provide effective analgesia after a variety of surgical procedures either by bolus ${ }^{11.35 .36}$ or bolus plus infusion ${ }^{37}$ Bolus doses range from 15 to $75 \mu \mathrm{g}$ and infusions $2-3 \mu \mathrm{g} \cdot \mathrm{kg}^{-1} \cdot \mathrm{hr}^{-1}$. Onset of analgesia is very rapid and may be related to uptake into the blood stream. ${ }^{38}$ Duration of analgesia, like fentanyl, is short with bolus doses $(3-6 \mathrm{hr})$. Severe respiratory depression is associated with the larger doses. "

\section{Other forms of acute pain}

Epidural opioids have been used successfully for analgesia in trauma patients especially in the presence of multiple fractured ribs (morphine), ${ }^{39}$ as an alternative to general anaesthesia or epidural local anaesthesia for extracorporeal shock wave lithotripsy (fentanyl), ${ }^{40}$ for pain associated with myocardial infarction (morphine), ${ }^{41}$ thrombophlebitis, herpes zoster and nephrolithiasis. ${ }^{39}$

\section{Epidural opioid/local anaesthetic combinations}

Recent interest in the combination of epidural opiates and local anaesthetic agents has shown that small doses of opioids added to dilute solutions of local anaesthetic agents can enhance and prolong analgesia. In obstetrical practice the following combinations have been shown to be effective: ${ }^{5}$ fentanyl $50 \mu \mathrm{g} / 0.125$ per cent bupivicaine, sufentanil $20-30 \mu \mathrm{g} / 0.125-0.25$ per cent bupivicaine, alfentanil $10 \mu \mathrm{g} \cdot \mathrm{kg}^{-1} / 0.125$ per cent bupivicaine and alfentanil $10 \mu \mathrm{g} \cdot \mathrm{kg}^{-1} \cdot \mathrm{hr}^{-1} / 0.125$ per cent bupivicaine $10 \mathrm{ml} \cdot \mathrm{kg}^{-1}$, butorphanol $2-3 \mathrm{mg} / 0.25$ per cent bupivicaine and morphine $2 \mathrm{mg} / 0.25$ per cent bupivicaine. Fentanyl and bupivicaine mixtures are also effective after surgery although larger doses $(200 \mu \mathrm{g})$ were necessary to prolong analgesia. ${ }^{42}$ Other opioids that have proved effective after various forms of surgery when combined with low-dose bupivicaine include diamorphine, ${ }^{43}$ sufentanil,$^{44}$ fentanyl, ${ }^{45}$ and morphine ${ }^{46}$ In general the use of low-dose opioid/dilute concentration local anaesthetic provides adequate analgesia with minimal side effects related to both the opioid and the local anaesthetic agent.

\section{Adverse effects of epidural opioids}

\section{NAUSEA AND VOMITING}

The incidence of nausea and vomiting appears to be higher in volunteers ${ }^{47}$ ( 50 per cent) given epidural morphine compared with the incidence in postoperative patients (17-30 per cent). ${ }^{2}$ Lipid soluble opioids seem to have a lower incidence of nausea and vomiting. ${ }^{2}$

\section{URINARY RETENTION}

Urinary retention occurs with an increased incidence in volunteers ${ }^{47}$ compared with postoperative patients (up to 40 per cent). ${ }^{3}$ Interestingly the urodynamic effects of epidural morphine are probably not dose-related. ${ }^{2}$ Lipid soluble opioids also cause urinary retention although the incidence is probably lower than with morphine. ${ }^{2}$

\section{PRURITIS}

Pruritus occurred in 100 per cent of volunteers given 10 mg of epidural morphine but only in 28 per cent of patients after surgery who were given a similar dose. ${ }^{2}$ With lower doses of epidural morphine the incidence of pruritus decreases dramatically. ${ }^{2}$ Pruritus also occurs with lipid soluble opioids but to a lesser degree. 


\section{RESPIRATORY DEPRESSION}

Respiratory depression and spinal opioids have been extensively reviewed. ${ }^{4}$ The true incidence of respiratory depression after epidural morphine is not known although life-threatening respiratory depression is uncommon. ${ }^{4}$ In general, after epidural opiods, respiratory depression is related to the dose, age, severity of concurrent illness, catheter placement, concomitant use of parenteral opioids or sedatives, and possibly change in position and coughing (increased intrathoracic pressure) ${ }^{2-4}$ The use of low-dose infusions may decrease the risk of respiratory depression. Morphine-related respiratory depression tends to follow spinal CSF circulation characteristics ${ }^{2,4}$ and thus the appearance of late respiratory depression occurs in some patients. With lipid soluble opioids respiratory depression has been reported less frequently ${ }^{4}$ although large doses are associated with this problem. ${ }^{11}$ Respiratory depression was seen very early after administration of lipid soluble opioids ${ }^{11,14}$ and thus may be related to vascular absorption with associated high serum concentration.

Other adverse effects include dysphoria (uncommon) and sedation. All the adverse effects can be reversed with small doses of naloxone. ${ }^{2}$

\section{Outcome studies related to epidural opioids}

Several studies have been performed which indicate that epidural opioids produce a decrease in overall morbidity and mortality when compared to other methods of postoperative pain relief. ${ }^{18.48 .49}$ As a result, the judicious use of epidural opioids for the control of acute pain in selected patients has become a powerful tool for the acute pain therapist.

\section{References}

1 Gregg $R$. Spinal analgesia, in management of postoperative pain. Anesthesiology Clinics of North America 1989; 7: 79-100.

2 Cousins MJ, Cherry DA, Gourlay GK. Acute and chronic pain: use of spinal opioids. In Cousins MJ, Bridenbaugh PO (Eds.). Neural Blockade. Clinical Anaesthesia and Management of Pain. 2nd ed., Philadelphia: J.B. Lippincott Co., 1988; 955-1029.

3 Morgan $M$. The rational use of intrathecal and extradural opioids. Br J Anaesth 1989; 63: 165-88.

4 Etches $R C$, Sandler $A N$, Daley $M D$. Respiratory depression and spinal opioids. Can J Anaesth 1989; 36: 165-85.

5 Shnider SM. Epidural and subarachnoid opiates in obstetrics. ASA Refresher Course 1989; 225.

6 McQuay HJ, Sullivan AF, Smallman K, Dickenson AH. Intrathecal opioids, potency and lipophilicity. Pain 1989; 36: 111-5.
7 Nordberg G, Hedner T, Mellstrand T, Dahlstrohm B. Pharmacokinetic aspects of epidural morphine analgesia. Anesthesiology 1983; 58: 545-51.

8 Sjostrom S, Hartvig P, Persson MP. Tamsen A. Pharmacokinetics of epidural morphine and meperidine in humans. Anesthesiology 1987; 67: 877-88.

9 El-Baz NM, Faber LP. Jensik RJ. Continuous epidural infusion of morphine for treatment of pain after thoracic surgery: a ncw tcchnique. Anesth Analg 1984; 63: 757-64.

10 Gourlay GK, Cherry DA, Plummer JL, Armstrong PJ. Cousins $M J$. The influence of drug polarity on the absorption of opioid drugs into CSF and subsequent cephelad migration following lumbar epidural administration: application to morphine and pethidine. Pain 1987; 31: 297-305.

11 Whiting WC, Sandler AN, Lau LC et al. Analgesic and respiratory effects of epidural sufentanil in patients following thoracotomy. Ancsthesiology 1988; 69: 36-43.

12 Hasenbos $M$, Gielen M, Bos J, Tielbeek E, Stanton-Hicks $N$, Van Egmond J. High thoracic epidural sufentanil for post thoracotomy pain. Influence of epinephrine as an adjuvant. A double-blind study. Anesthesiology 1988; 69: 1017-22.

13 Loper KA, Ready LB, Downey $M$ et al. Epidural and intravenous fentanyl infusions are clinically equivalent following knee surgery. Anesth Analg 1989; 70: 72-5.

14 Badner NH, Sandler AN, Koren G, Lawson S, Klein J, Einarson $T R$. Lumbar epidural fentanyl infusions for post-thoracotomy patients: analgesic, respiratory, and pharmacokinetic effects. J Cardiothoracic Anesth 1990 (in press).

15 Panos L, Sandler AN, Stringer DG, Badner N, Lawson S, Koren $G$. Continuous infusions of lumbar epidural fentanyl and intravenous fentanyl for postthoracotomy pain relief: I. analgesic and pharmacokinetic effects. Can J Anaesth 1990 (in press).

16 Martin S, Salbaing J, Blaise G, Tetrault L. Epidural morphinc for postoperative pain relief: a dose-response curve. Anesthesiology 1982. 56: 423-6.

17 Crawford RD, Batra MS, Fox F. Epidural morphine dose response for postoperative analgesia. Anesthesiology 1981; 55: A150.

18 Shulman M, Sandler AN, Bradley JW, Brebner J, Young $P S$. Post-thoracotomy pain and pulmonary function following epidural and systemic morphine. Ancsthesiology 1984; 61: 569-75.

19 Dahlstrom $B$. Pharmacokinetics and pharmacodynamics of epidural and intrathecal morphine. Int Anesthesiol Clin 1986; 24: 29-42.

20 Fromme GA, Steidl LJ, Danielson DR. Comparison of lumbar and thoracic epidural morphine for relief of postthoracotomy pain. Anesth Analg 1985; 64: 454-5.

21 Grant GJ, Zakowski M, Ramanathan S, Turndorf H. Lumbar versus thoracic administration of epidural morphine 
for post-thoracotomy analgesia. Anesth Analg 1990; 70: S134.

22 Etches $R C$. Sandler $A N$. Analgesic effects of epidural nalbuphine in post-thoracotomy patients. Can J Anaesth, 1989; 36: S156-7.

23 Gurel A, Unal, Elevli M, Eren E. Epidural morphine for postoperative pain relief in anorectal surgery. Anesth Analg 1986; 65: 499-502.

24 Rutberg $H$, Hakanson E, Erberg B, Jorfeldt $L$, Martensson $J$, Schildt $B$. Effects of extradural administration of morphine or bupivicaine on the endocrine response to upper abdominal surgery. Br J Anaesth 1984; 56: 233-8.

25 Rosen MA, Hughes SC, Shnider SM et al: Epidural morphine for the relief of postoperative pain and cesarean delivery. Anesth Analg 1983, 62; 666-72.

26 Daley MD. Sandler AN, Turner KE, Vosu H, Slavchenko $P$. A comparison of epidural and intramuscular morphine in patients following cesarean section. Anesthesiology 1990; 72: 289-94.

$27 E l-B a z N$, Goldin M. Continuous epidural infusion of morphine for pain relief after cardiac operations. J Thorac Cardiovase Surg 1987; 93: 878-83.

28 Melendez J, Cirella V, Dodds $T$. Lumbar epidural analgesia post-thoracic surgery. Anesth Analg 1988; 67: S147.

29 Naulty JS, Datta S, Ostheimer GW, Johnson MD, Burger $G A$. Epidural fentanyl for postcesarean delivery pain management. Anesthesiology 1985; 63: 694-8.

30 Wolfe $M J$. Davies $G K$. Analgesic action of extradural fentanyl. Br J Anaesth 1980; 52: 357-8.

31 Renaud B, Brichant $J F$, Clergue $F$, Chauvin $M$, Levron $J C$, Viars $P$. Ventilatory effects of continuous epidural infusion of fentanyl. Anesth Analg 1988; 67: 971-5.

32 Bailey $P W$, Smith $B E$. Continuous epidural infusion of fentanyl for postoperative analgesia. Anesthesia 1980; 35: 1002-6.

33 Badner NH, Sandler AN, Leitch L, Koren G. Analgesic and respiratory effects of continuous lumbar cpidural fentanyl in post-thoracotomy patients. Can J Anacsth. 1989; 36: S69.

34 Siringer DG, Sandler AN, Panos L, Lawson S, Einarson $T R, B a d n e r N$. Continuous infusions of lumbar epidural fentanyl and intravenous fentanyl for postthoracotomy pain relief: II. Respiratory effects. Can J Anaesth 1990; 37 (in press).

35 Donadoni $R$, Rolly $G$, Noorduin $H$, Vanden Busche $G$. Epidural sufentanil for postoperative pain relief. Anaesthesia 1985; 40: 634-38.

36 Cohen $S E$, Tan $S$, White $P F$. Sufentanil analgesia following cesarean section: epidural versus intravenous administration. Anesthesiology 1988; 68: 129-34.

37 Cheng $Y$, Koebert RF, Hopwood M, Stommel KA, Roerhig $D, K a y J$. Continuous epidural sufentanil infusion for post-operative analgesia. Anesthesiology 1987, 67: A233.
38 Koren $G$, Sandler AN, Klein J et al. Relationship between the pharmacokinetics and the analgesic and respiratory pharmacodynamics of epidural sufentanil. Clin Pharmacol Ther 1989; 46: 458-62.

39 Magora $F$, Olshwang D, Eimerl $D$ et al. Obscrvations of extradural morphine analgesia in various pain conditions. $\mathrm{Br} \mathrm{J}$ Aanacsth 1980; 52: 247-52.

40 Pandit SK, Powell RB, Crider B, McLarem ID, Rutter $T$. Epidural fentanyl: a simple and novel approach to anaesthetic management for extracorporeal shock wave lithotripsy (ESWL). Anesthesiology 1985; 67: A225.

41 Skoeld $M$, Gillberg $L$, Ohlsson $O$. Pain relief in myocardial infarction after continuous epidural morphine analgesia. New Engl J Med 1985; 312: 650.

42 Rucci FS, Cardamone M, Migliori P. Fentanyl and bupivicaine mixtures for extradural blockade. $\mathrm{Br} \mathrm{J}$ Anaesth 1985; 57: 275-84.

43 Lee A, Simpson D, Whitfield A, Scott DB. Postoperative analgesia by continuous extradural infusion of bupivicaine and diamorphine. Br J Anacsth 1988; 60: 845-50.

44 Zwarts SJ, Hasenbos MAMW, Gielen MJM, Kho HG. The effect of continuous cpidural analgesia with sufentanil and bupivicaine during and after thoracic surgery on the plasma cortisol concentration and pain relief. Rcg Anesth $1989 ; 14: 183-8$.

45 Tewes $P A$, Vella $L H$, Thomas $S$, Goll HM. Epidural fentanyl and bupivicaine combinations in patients undergoing pelvic surgery. Anesthesiology 1988; 69: Al06.

46 Gregg RV, Denson DD, Knarr DC, Stuebing RC. Continuous epidural infusions of bupivicaine and morphine versus systemic narcotic analgesics for postoperative pain relief. Ancsthesiology 1988; 69: A384.

47 Bromage PR. Camporesi E, Durant PA, Nielsen CH. Rostral spread of epidural morphine. Anesthesiology 1982; 56: 431-6.

48 Yeager MP, Glass DD, Neff RK, Brinck-Johnsen $T$. Epidural anesthesia and analgesia in high-risk surgical patients. Ancsthcsiology 1987; 66: 729-36.

49 Rawal N, Sjostrand U, Christofferson E, Dahlstrom B. Arvill A, Rydman $H$. Comparison of intramuscular and epidural morphine for postoperative analgesia in the grossly obese: influence on postoperative ambulation and pulmonary function. Anesth Analg 1984; 63: 8-14. 


\section{Traitement de la dou- leur aiguë par analgésie épidurale aux opiacés}

L'apparition de l'analgésie rachidienne s'est accompagnée d'une explosion de publications et de revues substantielles. $^{1-4}$ L'approche épidurale avec narcotiques est l'approche la plus populaire pour une variété d'indications, à cause de la facilité avec laquelle on peut obtenir l'analgésie prolongée, soit par une infusion ou par bolus répétés. Cette approche présente moins de complications que la voie sous-arachnoïdienne. ${ }^{3}$ Cette revue s'adressera à l'approche épidurale avec narcotiques de l'analgésie pour douleur aigüe.

Utilisation pour analgésie épidurale avec narcotiques Les indications mentionnées pour les narcotiques épiduraux dans le contrôle de la douleur aigüe ${ }^{2}$ comprennent la période péri-opératoire, post-opératoire (thoracotomie, chirurgie orthopédique, prostatectomie, chirurgie générale et abdominale, chirurgie gynécologique, césarienne), les avortements du second trimestre, la douleur post-traumatique et la douleur associée à des conditions médicales (infarctus du myocarde, thrombophlébite, herpes, néphrolithiase). Même s'il y a plusieurs travaux où l'on a rapporté l'utilisation d'opiacés par voie épidurale pour soulager la douleur pendant la travail, ${ }^{2}$ la plupart de ceux-ci mentionnent une analgésie insuffisante même si les opiacés par voie intrathécale étaient plus efficaces. ${ }^{5} \mathrm{La}$ liste des opiacés considérés efficaces par voie épidurale comprend : morphine, nicormorphine, hydromorphone, diamorphine, méthadone, mépéridine, buprénorphine, fentanyl, sufentanil, alfentanil, lofentanil, nalbuphine, butorphanol et pentazocine.

\section{Site d'action de l'analgésie rachidienne}

Les résultats de nombreux travaux chez l'animal indiquent que la corne postérieure de la moëlle est le site d'action primaire des opiacés. ${ }^{2}$ Ils exercent vraisemblablement une action pré- et post-synaptique d'inhibition de la transmission afférente primaire dans la moelle. Les opiacés agissent au niveau des récepteurs mu, delta et kappa $(40,10$ et 50 pour cent respectivement des récepteurs médullaires aux opiacés). Au niveau neuronal les mécanismes sont complexes mais vraisemblablement de nature inhibitrice, et probablement fonctionnent par l'intermédiaire de canaux ioniques (les récepteurs mu et delta sont associés à des canaux potassiques calcium dépendants ou voltage dépendants; les kappa inhibent les canaux calciques voltage-dépendants). Dans la douleur aigüe, la douleur incisive (fibres A-delta) est moins bien contrôlée que la douleur sourde (fibres-C). Ceci peut être dû à la localisation profonde des fibres A-delta dans la corne postérieure comparativement aux fíbres- $\mathrm{C}$, à la plus grande facilité d'atténuer la sommation temporale agissant à la membrane neuronale avec les fibres- $C$ à conduction lente comparativement au fibres-A-delta à conduction rapide, à la sélectivité de différents types de douleur pour certains récepteurs, et à des facteurs pharmacocinétiques qui déterminent la quantité d'opiacés qui atteignent les récepteurs.

\section{Pharmcocinétique des opiacés épiduraux}

Les opiacés épiduraux peuvent être administrés en bolus intermittents ou en combinaison bolus/infusion. Les profils pharmacocinétiques dans le sang, le liquide céphalo-rachidien et la moelle des différents opiacés sont complexes et reliés au mode d'administration (bolus ou infusion), à la dose administrée, la solubilité dans les lipides, la captation par la graisse épidurale et les structures lipidiques non spécifiques de la moelle. ${ }^{6}$ La morphine, un opiacé hautement ionisé et hydrophile, traverse la dure-mère lentement et apparait dans le liquide céphalo-rachidien en 15 minutes avec une concentration maximale de 90 à 120 minutes après l'administration.' Cependant, les pics de concentration plasmatiques sont constatés après 15 minutes et les courbes plasmatiques sont similaires à celles obtenues après une injection intramusculaire. Quatre vingt pour cent de la morphine est toujours présente dans le liquide céphalo-rachidien quatre heures après l'injection, et 50 pour cent après 12 heures. $^{8}$ Ceci confirme le long temps de latence et la durée prolongée de l'analgésie après l'administration de morphine épidurale en bolus. Le profil pharmacocinétique d'une infusion continue épidurale de morphine a fait l'objet de moins de travaux mais était associé à une analgésie adéquate et des niveaux sériques non détectables de morphine dans une étude. ${ }^{27}$ Par contre les opiacés liposolubles (quand même ionisés) tels la mépéridine pénètrent dans le liquide céphalo-rachidien rapidement même si la captation plasmatique de cette dernière est plus lente que celle de la morphine. ${ }^{10}$ Les opiacés hautement solubles tels le fentanyl et le sufentanil sont rapidement absorbés par l'espace vasculaire lorsque donnés en bolus 
ou en infusion, et peuvent produire certains de leurs effets analgésiques via cette absorbtion systémique. ${ }^{11-15}$ Il n'y a pas d'étude chez l'homme sur la pharmacocinétique neuraxiale des opiacés hautement liposolubles.

\section{Effets analgésiques}

\section{Douleur post-opératoire}

Les doses requises dépendent de la procédure chirurgicale, des opiacés choisis, de l'emplacement du cathéter, de l'utilisation de bolus seul ou de bolus avec infusion, de l'âge, de la sévérité de la condition médicale, de l'utilisation d'agent associés (e.g., anesthésiques locaux), et de la variabilité de la réponse entre patients. ${ }^{2,3} \mathrm{La}$ comparaison de certains opiacés peu solubles dans les lipides (e.g., morphine) et d'opiacés hautement liposolubles (e.g., fentanyl, sufentanil) illustre les effets différents de ces deux classes de substances lorsqu'utilisées dans l'espace épidural.

\section{Morphine}

Même si la morphine épidurale est largement utilisée, il y a peu d'études qui en évaluent les doses. Pour la chirurgie des membres inférieurs, des bolus de 2 à $5 \mathrm{mg}$ sont efficaces. ${ }^{16} \mathrm{La}$ chirurgie abdominale et thoracique semble demander des doses plus élevées lorsque les cathéters sont en position lombaire $(5-10 \mathrm{mg}) .{ }^{17,18}$ Le début d'action tend à être lent et peut prendre jusqu'à 60 minutes à survenir. ${ }^{16,19}$ Les travaux évaluant l'effet de la position du cathéter (lombaire vs thoracique) pour l'analgésie thoracique ont produit des résultats controversés ${ }^{20.21}$ même si le début de l'analgésie et la quantité de substance nécessaire peuvent être diminués avec les cathéters en position thoracique. ${ }^{21}$ De plus, les doses requises peuvent être moins importantes si la morphine épidurale est donnée avant l'intervention ou très tôt pendant la chirurgie. ${ }^{22-24}$ On a noté une analgésie prolongée avec de petites doses (3-5 mg) après les césariennes. ${ }^{25,26} \mathrm{On}$ a rapporté aussi que des infusions de morphine de l'ordre de 0,1 à 0,2 $\mathrm{mg} \cdot \mathrm{hre}^{-1}$ après une bolus de $2 \mathrm{mg}$ ont aussi été efficaces. ${ }^{9.27}$

\section{Opiacés liposolubles}

\section{FENTANYL}

Le fentanyl épidural a été utilisé pour plusieurs types de douleur post-operatoire, autant en bolus ${ }^{28-30}$ qu'en combinaison avec une infusion. ${ }^{14,15.31 .32}$ Le début de l'analgésie est rapide (5-20 min) mais de courte durée (2-4 hre), si une infusion n'est pas débutée. La nécessité d'utiliser des doses importantes en infusion $\left(\mathrm{I}-2 \mu \mathrm{g} \cdot \mathrm{kg}^{-1} \cdot \mathrm{hre}^{-1}\right)$ en particulier avec des cathéters en position lombaire, après chirurgie thoracique ${ }^{14,15.33}$ peut être dûe à la captation rapide du fentanyl épidural dans le flot sanguin ${ }^{14,15,33}$ et par des sites de liaison non spécifiques dans la moelle. ${ }^{6} \mathrm{ll}$ y a de plus en plus d'information accumulée qui indique que les infusions épidurales et intraveineuses de fentanyl produisent une analgésie équivalente et présentent un incidence similaire d'effets secondaires. ${ }^{13,15,34}$

\section{SUFENTANIL}

Le sufentanil épidural a été utilisé pour amener une analgésie efficace après un éventail de procédures chirurgicales, soit par bolus ${ }^{11,35.36}$ ou en bolus associé à des infusions. ${ }^{37}$ Les doses en bolus varient de 15 à $75 \mu \mathrm{g}$ et les infusions de 2-3 $\mu \mathrm{g} \cdot \mathrm{kg}^{-1} \cdot \mathrm{hre}^{-1}$. Le début de l'analgésie est très rapide et peut être relié à la captation dans la circulation. ${ }^{38}$ La durée de l'analgésie, comme celle du fentanyl, est courte avec les bolus (3-6 hre). Des doses importantes s'accompagnent de dépression respiratoire sévère. ${ }^{11}$

\section{Autres formes de douleur aigüe}

Les opiacés par voie épidurale ont été utilisés avec succès pour l'analgésie chez les patients traumatisés, surtout en présence de fractures de côtes multiples (morphine), ${ }^{39}$ comme alternative à l'anesthésie générale ou épidurale avec anesthésiques locaux pour la lithotripsie (fentanyl), ${ }^{40}$ pour la douleur associée à l'infarctus (morphine), ${ }^{41}$ la thrombophlébite, l'herpes zoster et la néphrolithiase. ${ }^{39}$

\section{Combinaison d'opiacés et d'anesthésiques locaux par voie épidurale}

L'intérêt récent pour les combinaisons d'opiacés épiduraux et d'anesthésiques locaux a montré que de petites doses d'opiacés ajoutées à des solutions diluées d'anesthésiques locaux peuvent augmenter et prolonger l'analgésie. En pratique obstétricale les combinaisons suivantes ont été jugées efficaces : 5 fentanyl $50 \mu \mathrm{g}$ /bupivacaïne 0,125 pour cent, sufentanil $20-30 \mu \mathrm{g}$ /bupivacaïne $0,125-0,25$ pour cent, alfentanil $10 \mu \mathrm{g} \cdot \mathrm{kg}^{-1}$ bupivacaïne 0,125 pour cent et alfentanil $10 \mu \mathrm{g} \cdot \mathrm{kg}^{-1} \cdot \mathrm{hre}^{-1}$, bupivacaïne à 0,125 pour cent à $10 \mathrm{ml} \cdot \mathrm{hre}^{-1}$, butorphanol 2-3 mg/bupivacaine 0,25 pour cent et morphine 2 mg/bupivacaïne 0,25 pour cent. Les combinaisons de bupivacaïne et fentanyl sont aussi efficaces après la chirurgie malgré que des doses plus importantes $(200 \mu \mathrm{g})$ aient été nécessaires pour prolonger l'analgésie. ${ }^{42} \mathrm{D}$ 'autres opiacés, jugés efficaces après plusieurs types de chirurgies, quand ils ont été combinés avec la bupivacaïne à faible dose, incluent la diamorphine ${ }^{43}$ le sufentanil, ${ }^{44}$ le fantanyl,$^{45}$ et la morphine. ${ }^{46}$ En général l'utilisation de petites doses d'opiacés associés à des concentration diluées d'anesthésiques locaux donnent une analgésie adéquate avec des effets secondaires minimaux associés autant aux opiacés qu'à l'anesthésique local. 


\section{Effets secondaires des opiacés épiduraux}

\section{NAUSEEE ET VOMISSEMENTS}

L'incidence de nausées et vomissements semble être élevée chez les volontaires (50 pour cent) qui ont reçu de la morphine épidurale lorsque que comparée à celle des patients en post-opératoire (17-30 pour cent). ${ }^{2}$ Les opiacés liposolubles semblent démontrer une plus faible incidence de nausées et de vomissements. ${ }^{2}$

\section{RÉTENTION URINAIRE}

La rétention urinaire se produit avec une incidence plus élevée chez les volontaires ${ }^{47}$ lorsque comparés à des patients qui ont subi une chirurgie (jusqu'à 40 pour cent). ${ }^{3}$ Curieusement, les effets urodynamiques de la morphine épidurale ne blent pas avoir de relation avec la dose. ${ }^{2}$ Les opiacés liposolubles vont aussi causer de la rétention urinaire mais l'incidence est plus faible qu'avec la morphine. $^{2}$

\section{LE PRURIT}

Le prurit se produit chez 100 pour cent des volontaires qui reçoivent $10 \mathrm{mg}$ de morphine épidurale mais seulement chez 28 pour cent des patients qui ont reçu une dose semblable. ${ }^{2}$ Avec des doses plus faibles de morphine épidurale l'incidence de prurit diminue de façon importante. $^{2}$ Le prurit se produit aussi avec les opiacés liposolubles mais à un degré moindre.

\section{DÉPRESSION RESPIRATOIRE}

L'association d'opiacés rachidiens et de dépression respiratoire a été revue récemment de façon extensive. ${ }^{4}$ L'incidence véritable de dépression respiratoire après la morphine épidurale n'est pas connue ; par contre, la dépression respiratoire menaçante est peu fréquente. ${ }^{4}$ En général, après les opiacés épiduraux, la dépression respiratoire est en relation avec la dose, l'âge, la sévérité de la condition médicale, la position du cathéter, l'usage concomittant d'opiacés ou de sédatifs parentéraux, et possiblement des changements de position et la toux (augmentation de la pression intra-thoracique) ${ }^{2-4}$ L'utilisation d'infusions à faible dose peut diminuer le risque de dépression respiratoire. La dépression respiratoire associée à la morphine tend à aller de pair avec les caractéristiques de la circulation du liquide céphalorachidien $^{2,4}$ et ainsi l'apparition de dépression respiratoire tardive se produit chez certains patients. Avec les opiacés liposolubles, la dépression respiratoire a été mentionnée moins fréquemment ; ${ }^{4}$ par contre des doses importantes sont associées avec ce problème. " La dépression respiratoire est apparue très tôt après l'administration d'opiacés liposolubles ${ }^{11,14}$ et ainsi peut être en rapport avec l'ab- sorption vasculaire, elle-même associée à des concentrations sériques élevées.

Les autres effets secondaires comprennent la dysphorie (peu courante) et la sédation. Tous les effets secondaires peuvent être contrecarrés par de petites doses de naloxone. ${ }^{2}$

Études d' issues (outcome) associées aux opiacés épiduraux De nombreuses études indiquent que l'épidurale aux narcotiques produit une diminution globale de morbidité et mortalité, lorsque comparée à d'autres méthodes d'analgésie post-opératoire. ${ }^{18,48,49}$ En conséquence, l'utiilisation judicieuse d'opiacés épiduraux pour le contrôle de la douleur aigüe chez des patients choisis est devenue un outil puissant pour le traitement de la douleur aigüe.

\section{Références \\ (Voir page Sxxxv)}

